\title{
Meeting Summary: A 2017 View of Active Galactic Nuclei
}

\author{
Hagai Netzer* \\ School of Physics and Astronomy, Tel Aviv University, Tel Aviv, Israel
}

The topics covered in this summary review reflect the major areas discussed in the Padova meeting, in April 2017. They are divided into general categories: those areas where large progress has been made leading to a real new understanding (what we are doing "right"), and those where we are still in the dark (what we are doing "wrong"). The division reflects the status of the field as well as my subjective opinion.

Keywords: black holes, star formation, host galaxies, cosmology, accretion disks, reverberation mapping, AGN-feedback

\section{INTRODUCTION: "RIGHT" AND "WRONG" IN AGN STUDY}

The topics covered in this meeting are very broad: from the central black hole $(\mathrm{BH})$ and the accretion disk (AD), to the observations and physics of the broad line region (BLR), narrow line region (NLR), AGN-tori, AGN winds and feedback, the various types of AGN, the connection

OPEN ACCESS

Edited by: Mauro D'Onofrio, Università degli Studi di Padova, Italy

Reviewed by: Luka C. Popovic, Astronomical Observatory, Serbia Gordon Richards, Drexel University, United States

*Correspondence: Hagai Netzer netzer@wise.tau.ac.il

Specialty section: This article was submitted to Milky Way and Galaxies, a section of the journal Frontiers in Astronomy and Space Sciences

Received: 15 September 2017 Accepted: 12 March 2018 Published: 09 May 2018

Citation: Netzer H (2018) Meeting Summary: A 2017 View of Active Galactic Nuclei.

Front. Astron. Space Sci. 5:10. doi: 10.3389/fspas.2018.00010 to the host galaxy and its star forming (SF) regions, and even AGN in a cosmological context. To summarize this huge area (usually divided into sub-areas and discussed in more specialized meetings) I chose to assess the quality of the observations presented here, and the complexity and reality of the associated models, by assigning to each one of the categories one of two "quality flags," either "right," or "wrong." These should not be understood in their every-day meaning. Rather, they reflect my personal view of where we have made big progress, and are starting to understand the big picture, and where we are probably missing some essential points, and hence may be proceeding in the wrong direction. Thus, the meaning of "we must be doing something wrong" used often in this review, means that in my opinion, we are missing some important ingredients either because we misunderstand the objects or phenomena we are studying, or because the work necessary to reach a real understanding is so complicated, or time-consuming, that it has not yet been done. Obviously there is no way to refer to all the talks and posters presented in the meeting, many of which represent excellent work. I only chose a small number of those that seem to represent the topics where "right" or "wrong," as defined here, are more clearly separable.

\section{THE AGN FAMILY: NEW MULTI-WAVELENGTH OBSERVATIONS} \subsection{Something Right: Systematic Study of LINERS, Seyferts, High Luminosity AGN and Objects Containing Disk-Like BLRs}

There is rapid progress in obtaining improved observations of large and small AGN samples. The SDSS is leading this field because of the very large number of sources observed spectroscopically in this survey. However, smaller samples, especially those that are carefully selected in one wavelength band and then studied in others, provide valuable information in terms of being more complete. The COSMOS sample is one such sample, alongside with the older PG-Quasar sample, the new BAT sample, and more. Examples of this type were provided by Márquez et al. (2017, LINERs and Seyfert 2s) and Richards (2017, type-I AGN in the SDSS sample combined with a new division 
into eigenvector groups), (Lusso and Risaliti, 2018) who provided a fresh look at X-ray sources and even some GAIA results that are going to contribute their part to the field in the near future (Angello, 2017). A somewhat different example, presented by Storchi-Bergmann et al. (2017), demonstrated the great advantage of looking at the rare group of double-hump broad line sources where studying a large sample in a very systematic way, leads to a new insight.

\subsection{Something Wrong: A Complete Picture of Radio AGN}

The clear and comprehensive review by Padovani (2017), as well as several talks on Blazars, only helped to demonstrate that we are still far from understanding many of these sources, their physics, variability and, most importantly, the connection (if any) between radio and optical-UV properties of many radio loud AGN. The Blazar field is perhaps the least understood and the new big observatories which help to extend their study to very high energies, and help to follow their variations in more detailed ways, seems to provide only incremental improvements over what was known 10 and 20 years ago. We must be doing something wrong in this area.

\section{MAPPING AND MODELING THE BLR AND MEASURING BH MASS}

\subsection{Something Right: Reverberation Mapping in One and Two Dimensions}

A giant leap forward in terms of 2D (location and kinematics) reverberation mapping (RM) of the BLR is the recent (20132014) study of NGC 5548 by HST and a host of ground-based telescopes. Despite being only one source, that may not be typical with respect to its very high X-ray luminosity compared with the optical-UV luminosity, this is a superb example of the power of well planned spectroscopic observations. The light-curves of different emission lines at various gas velocities are text-book examples which will likely provide the tool to solve some of the issues related to the physics of the BLR. The new information about time-dependent broad and narrow absorption lines, and the accretion disk itself, is equally exciting. Some examples of this were shown by Horne (2015) and Fausnaugh et al. (2017). The same group, now with the help of many others, is making plans to carry out a similar study of other AGN.

\subsection{Something Right: The R-L Relationship and Single-Epoch Mass Measurements}

Related to this is the impressive collection of sources (more than 70) where the emissivity weighted radius of the broad $\mathrm{H} \beta$ line has been measured through the correlated line and continuum variations, and variability may even be related to eigenvector 1 (Ilić et al., 2017; Bon et al., 2018). In some of the mapped sources, the $\mathrm{H} \beta$ line can be divided into several velocity bins allowing one to answer a simpler question of whether the BLR motion is mostly outflow, inflow, or closer to a bound rotational motion (Pancoast et al., 2014). Surprisingly, there is no single canonical dynamical pattern. Other important new results connect the $\mathrm{H} \beta$ emission region size to the Eddington ratio of the accreting $\mathrm{BH}$.

New, long expected RM results applied to the most luminous AGN, at high redshift, were presented by Kaspi et al. (2017) and Lira et al. (2018). They show that the CIV $\lambda 1549$ and Ly $\alpha$ emitting regions are much closer to the central $\mathrm{BH}$ compared with the $\mathrm{H} \beta$ emitting region, by a factor of $\sim 3$. The two RM campaigns that finally answered this question took $10-15$ years (!!) to completea good example showing what science can reveal when managing to convince large telescope TACs that some problems require more than a decade of observations. The other very good news in this area is the completion of VLT/GRAVITY that is capable of measuring, directly, BLR sizes in a small number of nearby type-I AGN.

Another idea, which is clearly becoming more visible due to the fact that more and more type-I AGN are studied, spectroscopically, in more and more detail, is the suggestion, by Marziani et al. (2018) that different locations in the eigenvector 1 plane hint to fundamentally different physics.

\subsection{Something Wrong: Phase-Space Modeling of the BLR}

Despite the impressive 2D RM maps of NGC 5548 presented in the meeting, and lower quality ones available for other sources, there is little if any progress in constructing consistent spatially and dynamically connected maps for the best studied source. We seem to be facing the same questions that were asked some 20 years ago, after the analysis of the very first optical-UV RM study of the same sources with ground based telescopes and the IUE satellite. This field is eagerly waiting for a more detailed models which will justify the huge resources invested in it.

Some good news in this area, not directly related to the mapping of the BLR, is a new model presented by Czerny et al. (2017), where the origin (and hence location) of the BLR clouds is the dusty outer parts of the central accretion disk. This and similar models can be tested by next generation 2D BLR models. Some other ideas that were proposed but never tested properly are related to the commonly assumed relationship $R_{B L R} \propto L^{\alpha}$ where $R_{B L R}$ is the BLR radius estimated from RM campaign, $L$ the monochromatic continuum at a chosen wavelength, and $\alpha=0.5 \pm 0.1$. Is it possible, in view of the dependence on the Eddington ratio discovered recently, and hence the role of radiation pressure force, that an alternative approximation of the type $R_{B L R}=c_{1} L^{\alpha}+c_{2}(L / M)$ will provide better $\mathrm{BH}$ mass estimates?

\subsection{Better Calibration of BH Masses}

While the commonly used single-epoch (SE) $\mathrm{BH}$ mass measurements is a substantial step forward, which is well recognized by the community, the field is still looking for a way to improve the calibration of these measurements, i.e., the factor $f_{B L R}$ in the expression $M_{B H}=f_{B L R} R_{B L R} V_{B L R}^{2} / G$ The way used by most researchers is based on the well known $M_{B H}-\sigma *$ relationship which provides the calibration of $f_{B L R}$ in the local universe, i.e., mostly for low luminosity AGN. A new promising method was presented by Mejía-Restrepo et al. (2018, paper presented by Lira). In this method, applied so far 
to high luminosity, high redshift $(z \sim 1.5)$ AGN, the observed FWHM of the broad lines can provide the desired calibration, i.e., $f_{B L R} \propto 1 / F W H M($ line $)$.

\section{ACCRETION DISKS AND DISK WINDS}

\subsection{Something Right: Thin Accretion Disk Models and the Optical-UV SED}

After years of study it is now apparent that the optical-UV continuum of at lease some AGN, those with large BH mass $\left(10^{8}-10^{9} \mathrm{M}_{\text {sun }}\right)$ and not too large Eddington ratio, can be adequately fitted by the canonical spectrum of a thin accretion disk. Apparently, the previous failure of such attempts was probably due to the lack of simultaneous observations that cover a large enough wavelength range.

\subsection{Something Wrong: The Spectrum and Properties of Slim Accretion Disks}

Despite heroic observational and theoretical efforts, slim accretion disks, those with an Eddingto ratio exceeding about 0.3, are not yet understood. The SED beyond the Lyman limit is not known observationally and there are clear discrepancies between theoretical predictions and the observed luminosity ratios of their NIR, extreme UV and X-ray radiation (Castelló-Mor et al., 2017).

\subsection{Disk Winds: Right and Wrong}

All theoretical slim disk models, as well as present-day (rather simplified) numerical simulations, show that strong disk winds must be present, especially close to the central $\mathrm{BH}$. The amount of accreted energy carried out in such winds, and hence not by radiation, is not known and the geometry and velocity fields can only be guessed. On a slightly larger scale, there was a lot of discussion about dusty and dust-free winds as presented by Elitzur (e.g., Elitzur and Netzer, 2016). In fact, it is hard to imagine a disk with a strong magnetic field that does not show this component. One claim is that the outflowing BLR clouds, and even the dusty torus, are also related to such flows.

Unfortunately, so far we do not have a clear and convincing observational evidence for such winds. The dynamical studies of the BLR gas only show the signature of outflows in a handfull of sources and other observations show exactly the opposite. There are however a couple of new observations that can be interpreted as a signature of a wind. The first is the "polar dust" found in a couple of sources studied with various interferometers (Hönig, 2016). The second, which I consider to be one of the most amazing results presented in the meeting, is the study of a source which is microlensed by a foreground star (Hutsemékers et al., 2017). Reconstructing the lens magnification map, allows one to combine location and velocity in the lensed BLR. It shows that the $\mathrm{H} \alpha$ line profile is produced by a flat, rotating BLR while the CIV $\lambda 1549$ line is probably produced in an outflowing polar gas. This way of probing the dynamics is extremely accurate but, unfortunately, can only be applied to a handful of lensed sources.

\section{STAR FORMATION GALACTIC-SCALE WINDS MERGERS AND FEEDBACK}

\subsection{Something Right: Observational Evidence for Outflow and Mergers}

In terms of new observations, and on-going effort, this topic is, arguably, the place where most observational effort has been made over the last few years. We have been presented with superb quality velocity maps, in different objects, showing large scale outward motion of ionized and molecular gas. The outflow can be associated with the vicinity of the $\mathrm{BH}$ (mostly X-ray outflows), the narrow line region, or even further away in the galaxy. Evidence comes from low redshift sources just "around the corner" and for redshift as large as 3-6. The available velocity maps are already of high quality and the coming new-generation IFU instruments, on JWST and several large ground-based telescopes, will no doubt provide even more detailed observations. ALMA is likely to play a role in proving, or disproving, the suggestion that many of the ionized outflow are associated with high mass-outflow rate molecular gas.

\subsection{Something Wrong: Interpreting Outflow and Feedback}

We are still in the dark regarding accurate measurements of mass outflow rates and hence the importance of AGNfeedback in quenching SF and in shaping the structure and evolution of the host galaxy. In ionized outflow, the main uncertainties are in those terms combining gas density and gas filling factor. The difficulty is due to poor observations (lack of outflow signature in several lines, not just the strongest ones) and the difficult estimate of the outflow filling factor. Molecular outflows are easier to model but questions about the production of $\mathrm{CO}$ lines in outflowing material still remain.

Present day feedback models are still not advanced enough to make clear and specific predictions about the galactic scale influence of the process. In particular, it is not very clear whether energy-conserved outflows, or momentum conserved outflows, are more important. One of them is associated with radiation pressure force mostly on dust grains. The other, with galactic scale shocked gas. Here, again, theory lags the observations, or perhaps more precisely, numerical simulations of galacticscale shocks, and feedback, have a long way to go. The larger scale, cluster-size feedback, associated with powerful radio jets and X-ray cavities, is perhaps better understood (Morganti, 2017).

Related questions are the correlation of SF and AGN luminosity in low and high redshift sources. Here, again, we heard some conflicting results reflecting the large uncertainties in measuring SF rates, mostly in the FIR, and in correlating it with optical and X-ray observations. Thus Ichikawa et al. (2017) showed several samples where $\mathrm{L}(\mathrm{AGN}) \propto \mathrm{L}(\mathrm{SF})$ while according to Stanley et al. (2015), such a correlation does not exist in any redshift.

The presence of near companions can be interpreted as indication for merger that can trigger $\mathrm{SF}$ and speed up the 
evolution of the system. The beautiful results presented by Fogasy et al. (2017), Kimball et al. (2015) and Trakhtenbrot et al. (2018) are quite amazing, given the redshift, size and brightness of the sources. However, they do not seem to be in agreement with each other, e.g., in relation to the question of which one is more FIR luminous, the AGN-host or the companion.

\section{COSMOLOGY WITH AGN}

\subsection{Something Wrong: All AGN-Based Methods}

Efforts to identify reliable tools based on AGN physics, that can help map the universe, and its expansion, at high redshift, and improve on the accuracy of present day measurements based on Type-Ia SNs, have not yet produced meaningful results. Some of these ideas have been mentioned in the meeting, e.g., the use of the observed luminosity of superEddington AGN, dust RM and its comparison with accurate IR interferometry (Hönig, 2016), the scaling of BLR density and ionization parameter (Negrete et al., 2017), or the relationship between X-ray and UV observations of type-I AGN (Lusso and Risaliti, 2018). Unfortunately, systematic uncertainties in all the methods, combined with a lack of understanding of some of the involved processes, prevent us from reducing the uncertainties of such methods to a trustable and useful level. We must be missing something very important in all these methods or, perhaps, AGN are simply not the tool to advance precision cosmology.

\section{OPTIMISTIC SUMMARY}

The approach followed in this summary review, of comparing things we understand ("we must be doing something right") with those we do not yet understand ("we must be doing something wrong") is useful since it allows us to produce an itemized list of

\section{REFERENCES}

Angello, A. (2017). Quasar lenses and galactic streams: outlier selection and Gaia multiplet detection. Month. Notices R. Astron. Soc. 471, 2013-2021. doi: 10.1093/mnras/stx1650

Bon, N., Bon, E., and Marziani, P. (2018). AGN broad line region variability in the context of eigenvector 1: case of NGC 5548. Front. Astron. Space Sci. 5:3. doi: $10.3389 /$ fspas.2018.00003

Castelló-Mor, N., Kaspi, S., Netzer, H., Du, P., Hu, C., Ho, L. C., et al. (2017). Unveiling slim accretion disc in AGN through X-ray and Infrared observations. Month. Notices R. Astron. Soc. 467, 1209-1221. doi: 10.1093/mnras/stx153

Czerny, B., Li, Y.-R., Sredzinska, J., Hryniewicz, K., Panda, S., Wildy, C., et al. (2017). Self-consistent dynamical model of the broad line region. Front. Astron. Space Sci. 4:5. doi: 10.3389/fspas.2017.00005

Elitzur, M., and Netzer, H. (2016). Disc outflows and high-luminosity true type 2 AGN. MNRAS 459, 585-594. doi: 10.1093/mnras/stw657

Fausnaugh, M. M., Peterson, B. M., Starkey, D. A., and Horne, K. (2017). the AGN STORM Collaboration. Continuum reverberation mapping of AGN accretion disks. Front. Astron. Space Sci. 4:55. doi: 10.3389/fspas.2017.00055

Fogasy J., Knudsen K. K., Lagos, C. D. P., Drouart, G., and Gonzalez-perez, V. (2017). On the frequency of star-forming galaxies in the vicinity of powerful AGNs: the case of SMM J04135+10277. Astron. Astrophys. 597:123. doi: 10.1051/0004-6361/201628173 topics, and hence specific goals, for future projects and meetings. Under the "right" header in this review I included: detailed and accurate new observations, larger and more complete samples, wider wavelength coverage, better spatial resolution, better time sampling, and more. The "wrong" category includes, in my opinion (and correct to 2017) topics like: understanding BH and galaxy evolution, simulating large scale baryonic processes like feedback, measuring mass outflow rates, understanding slim accretion disks and disk winds, proper modeling of the BLRs and dusty disk winds, and more. The common denominator is evident: We are making large and significant steps forward on the observational front but the theory, and the numerical simulations, lag behind. The optimistic view is that doing "wrong" is the first step for success. A nice summary of this idea is a clever quote from the writing of the late Susan Jeffers, a well known American psychologist and author, who once said: "If you haven't made any mistakes lately, you must be doing something wrong."

\section{AUTHOR CONTRIBUTIONS}

The author confirms being the sole contributor of this work and approved it for publication.

\section{FUNDING}

This research has been supported by the Israel Science Foundation grand 284/13.

\section{ACKNOWLEDGMENTS}

The information provided in this review is based on the excellent papers presented in this meeting, and the ideas and suggestions of my colleagues and students. The classification into the categories of right and wrong is entirely mine.

Hönig, S. F. (2016). Tori, discs, and winds: the first ten years of AGN interferometry. Astrophys. Space Sc. L. 439, 1-95. doi: 10.1007/978-3-319-39739-9 6

Horne, K. (2015). AGN Space Telescope and Optical Reverberation Mapping Project. IV. Velocity-Delay Mapping of Broad Emission Lines in NGC 5548. Available online at: http://adsabs.harvard.edu/abs/2015AAS...22510304H

Hutsemékers, D., Braibant, L., Sluse, D., Anguita, T., and Goosmann, R. (2017). New constraints on quasar broad absorption and emission line regions from gravitational microlensing. Front. Astron. Space Sci. 4:18. doi: 10.3389/fspas.2017.00018

Ilić, D., Shapovalova, A. I., Popovic, L. C., Chavushyan, V., Burenkov, A. N., Kollatschny, W., et al. (2017). Long-term monitoring of the broad-line region properties in a selected sample of AGN. Front. Astron. Space Sci. 4:12. doi: 10.3389/fspas.2017.00012

Ichikawa, K., Ricci, C., Ueda, Y., Matsuoka, K., Toba, Y., Kawamuro, T., et al. (2017). The complete infrared view of active galactic nuclei from the 70 month Swift/BAT catalog. Astrophys. J. 835:74. doi: 10.3847/1538-4357/835/1/74

Kaspi, S., Brandt, W. N., Maoz, D., Netzer, H., Schneider, D. P., and Shemmer, O. (2017). Reverberation mapping of high-luminosity quasars. Front. Astron. Space Sci. 4:31. doi: 10.3389/fspas.2017.00031

Kimball, A. E., Lacy, M., Lonsdale, C. J., and Macquart, J.-P. (2015). ALMA detection of a disc-dominated [C II] emission line at $z=4.6$ in the luminous QSO J1554+1937. MNRAS 452, 88-98. doi: 10.1093/mnras/stv1160 
Lira, P., Botti, I., Kaspi, S., and Netzer, H. (2018). Reverberation mapping of high-z, high-luminosity quasars. Front Astron Space Sci. 4:71. doi: $10.3389 /$ fspas.2017.00071

Lusso, E., and Risaliti, G. (2018). The physical relation between disc and coronal emission in quasars. Front. Astron. Space Sci. 4:66. doi: 10.3389/fspas.2017.00066

Márquez, I., Masegosa, J., González-Martin, O., Hernández-Garcia, L., Povic, M., Netzer, H., et al. (2017). The AGN nature of LINER nuclear sources. Front. Astron. Space Sci. 4:34. doi: 10.3389/fspas.2017.00034

Marziani, P., Dultzin, D., Sulentic, J. W., Del Olmo, A., Negrete, C. A., MartinezAldama, M. L., et al. (2018). A main sequence for quasars. Front. Astron. Space Sci. 5:6. doi: 10.3389/fspas.2018.00006

Mejía-Restrepo, J. E., Lira, P., Netzer, H., Trakhtenbrot, B., and Capellupo, D. (2018). The virial factor and biases in single epoch black hole mass determinations. Front. Astron. Space Sci. 4:70. doi: 10.3389/fspas.2017.00070

Morganti, R. (2017). The many routes to AGN feedback. Front. Astron. Space Sci. 4:42. doi: $10.3389 /$ fspas.2017.00042

Negrete, C. A., Dultzin, D., Marziani, P., Sulentic, J. W., Esparza-Arredondo, D., and Martïnez-Aldama, M. L. (2017). Quasars as cosmological standard candles. Front. Astron. Space Sci. 4:59. doi: 10.3389/fspas.2017.00059

Padovani, P. (2017). Active galactic nuclei at all wavelengths and from all angles. Front. Astron. Space Sci. 4:35. doi: 10.3389/fspas.2017.00035

Pancoast, A., Brewer, B. J., Treu, T., Park, D., Barth, A. J., Bentz, M. C., et al. (2014). Modelling reverberation mapping data II. Dynamical modelling of the Lick AGN Monitoring Project 2008 data set. Month. Notices R. Astron. Soc. 445, 3073-3091. doi: 10.1093/mnras/stu1419
Richards, G. (2017). Application of Independent Component Analysis to Legacy UV Quasar Spectra. Available online at: http://adsabs.harvard.edu/abs/2017hst.. prop15048R

Stanley, F., Harrison, C. M., Alexander, D. M., Swinbank, A. M., Aird, J. A., Del Moro, A., et al., (2015). A remarkably flat relationship between the average star formation rate and AGN luminosity for distant X-ray AGN. MNRAS 453, 591-604. doi: 10.1093/mnras/stv1678

Storchi-Bergmann, T., Schimoia, J. S., Peterson, B. M., Elvis, M., Denney, K. D., Eracleous, M., et al. (2017). Double-peaked profiles: ubiquitous signatures of disks in the broad emission lines of active galactic nuclei. 835:236. doi: 10.3847/1538-4357/835/2/236

Trakhtenbrot, B., Lira, P., Netzer, H., Cicone, C., Maiolino, R., and Shemmer, O. (2018). Fast-growing SMBHs in fast-growing galaxies, at high redshifts: the role of major mergers as revealed by ALMA. arXiv e-print arXiv:1801. 01508 .

Conflict of Interest Statement: The author declares that the research was conducted in the absence of any commercial or financial relationships that could be construed as a potential conflict of interest.

Copyright (C) 2018 Netzer. This is an open-access article distributed under the terms of the Creative Commons Attribution License (CC BY). The use, distribution or reproduction in other forums is permitted, provided the original author(s) and the copyright owner are credited and that the original publication in this journal is cited, in accordance with accepted academic practice. No use, distribution or reproduction is permitted which does not comply with these terms. 